OPEN ACCESS

Edited by:

Vincenzo Quagliariello, Istituto Nazionale Tumori Fondazione

G. Pascale, Scientific Institute for

Research, Hospitalization and

Healthcare (IRCCS), Italy

Reviewed by:

Yongbo Peng,

Chongqing Medical University, China

Xiang Zhao,

Huazhong University of Science

and Technology, China

Gang Zhao,

Beijing Hospital, China

*Correspondence:

Litu Zhang

zhanglitu@gmail.com

Kaisheng Liu

kaisheng_liu@163.com

${ }^{\dagger}$ These authors have contributed

equally to this work

Specialty section:

This article was submitted to Molecular and Cellular Oncology,

a section of the journal

Frontiers in Cell and Developmental

Biology

Received: 04 December 2020 Accepted: 04 October 2021

Published: 26 October 2021

Citation:

Zhou J, Wei W, Hou H, Ning S,

$L i \mathrm{~J}$, Huang $B$, Liu $K$ and Zhang $L$ (2021) Prognostic Value of C-Reactive

Protein, Glasgow Prognostic Score, and C-Reactive Protein-to-Albumin

Ratio in Colorectal Cancer.

Front. Cell Dev. Biol. 9:637650.

doi: 10.3389/fcell.2021.637650

\section{Prognostic Value of C-Reactive} Protein, Glasgow Prognostic Score, and C-Reactive Protein-to-Albumin Ratio in Colorectal Cancer

\author{
Jiahui Zhou't, Wene Wei't, Hu Hou ${ }^{2,3 t}$, Shufang Ning ${ }^{1}$, Jilin Li', Baoyue Huang', \\ Kaisheng Liu' ${ }^{2,3 *}$ and Litu Zhang ${ }^{1,4 *}$ \\ ${ }^{1}$ Department of Research, Affiliated Tumor Hospital, Guangxi Medical University, Nanning, China, ${ }^{2}$ Department of Laboratory \\ Medicine, Shenzhen People's Hospital, The First Affiliated Hospital, Southern University of Science and Technology, \\ Shenzhen, China, ${ }^{3}$ Department of Laboratory Medicine, Shenzhen People's Hospital, The Second Clinical Medical College, \\ Jinan University, Guangzhou, China, ${ }^{4}$ Guangxi Cancer Molecular Medicine Engineering Research Center, Nanning, China
}

Background: Emerging evidence suggests that inflammatory response biomarkers are predictive factors that can improve the accuracy of colorectal cancer (CRC) prognoses. We aimed to evaluate the prognostic significance of C-reactive protein (CRP), the Glasgow Prognostic Score (GPS), and the CRP-to-albumin ratio (CAR) in CRC.

Methods: Overall, 307 stage I-III CRC patients and 72 colorectal liver metastases (CRLM) patients were enrolled between October 2013 and September 2019. We investigated the correlation between the pretreatment CRP, GPS, and CAR and the clinicopathological characteristics. The Cox proportional hazards model was used for univariate or multivariate analysis to assess potential prognostic factors. A receiver operating characteristic $(\mathrm{ROC})$ curve was constructed to evaluate the predictive value of each prognostic score. We established CRC survival nomograms based on the prognostic scores of inflammation.

Results: The optimal cutoff levels for the CAR for overall survival (OS) in all CRC patients, stage I-III CRC patients, and CRLM patients were 0.16, 0.14, and 0.25, respectively. Kaplan-Meier analysis and log-rank tests demonstrated that patients with high CRP, CAR, and GPS had poorer OS in CRC, both in the cohorts of stage I-III patients and CRLM patients. In the different cohorts of CRC patients, the area under the ROC curve (AUC) of these three markers were all high. Multivariate analysis indicated that the location of the primary tumor, pathological differentiation, and pretreatment carcinoembryonic antigen (CEA), CRP, GPS, and CAR were independent prognostic factors for OS in stage I-III patients and that CRP, GPS, and CAR 
were independent prognostic factors for OS in CRLM patients. The predictors in the prediction nomograms included the pretreatment CRP, GPS, and CAR.

Conclusions: CRP, GPS, and CAR have independent prognostic values in patients with CRC. Furthermore, the survival nomograms based on CRP, GPS, and CAR can provide more valuable clinical significance.

Keywords: colorectal cancer, prognosis, C-reactive protein, Glasgow Prognostic Score, C-reactive protein-toalbumin ratio

\section{INTRODUCTION}

Colorectal cancer (CRC) is the third most common cancer and the second most common cause of cancer-related death worldwide. CRC is one of the most common gastrointestinal malignancies (Bray et al., 2018). The liver is the most common metastatic site of CRC, and the most common cause of death associated with CRC is liver metastasis (Hamilton et al., 2014). Surgery is still the primary curative modality for these patients. With increased treatment options, it has become increasingly important to choose the best treatment strategy. However, there is no reliable biomarker to identify stage I-III patients who are at high risk of poor prognosis and need to be treated with adjuvant chemotherapy. Regarding colorectal liver metastases (CRLM), there is a lack of reliable prognostic biomarkers to determine which patients will benefit from chemotherapy. Therefore, to achieve better clinical treatment outcomes, it is necessary to identify biomarkers that can better predict CRC prognoses.

Since Virchow first reported the association between inflammation and cancer in 1863, various studies have analyzed this relationship (Balkwill and Mantovani, 2001; Mantovani et al., 2008). Inflammation has been widely recognized as a factor that contributes to cancer progression, and many inflammatory markers, such as C-reactive protein (CRP), the Glasgow Prognostic Score (GPS), and the modified GPS (mGPS), have been shown to be associated with survival in CRC patients (Nasr et al., 2018; Matsubara et al., 2020). After curative treatment of CRC patients, CRP alone can be used as a strong prognostic indicator (Køstner et al., 2016; Matsubara et al., 2020). The GPS is a simple and valuable cumulative prognostic scoring system. It is based on two simple components-serum CRP and albumin-the levels of which are defined as normal or abnormal based on their laboratory reference ranges. Practitioners can divide cancer patients into three separate groups before treatment based on their GPS (McMillan et al., 2007). The CRP-to-albumin ratio (CAR) is based on a composite ratio. Recently, the value of the CAR in predicting the prognosis of CRC patients has been widely investigated (Ishizuka et al., 2016; Haruki et al., 2017). However, Climent et al. (2019) found that the difference in overall survival (OS) and disease-free survival between the high and low CAR

Abbreviations: CRC, colorectal cancer; CRLM, colorectal liver metastases; CEA, carcinoembryonic antigen; CA19-9, carbohydrate antigen 19-9; CAR, CRP/albumin ratio; CRP, C-reactive protein; GPS, Glasgow prognostic score; ROC, receiver operating characteristic; AUC, area under the ROC curve; $\mathrm{CI}$, confidence interval. groups was not significant; thus, the prognostic value of the CAR in CRC is still controversial.

Therefore, the present study aimed to assess the relationship between CRP, GPS, and CAR and the prognosis and clinicopathology of patients with CRC and to compare the prognostic value of these indicators in patients undergoing treatment for CRC.

\section{PATIENTS AND METHODS}

\section{Patients}

The data of 1,286 patients with CRC who were admitted to the Gastrointestinal Surgery Department of the Guangxi Medical University Cancer Hospital between October 2013 and September 2019 were retrospectively analyzed. Of these, 379 were included in this study and 907 were excluded because of incomplete medical information or lack of follow-up, the details of which are presented in Figure 1. All patients underwent scanning using abdominal B ultrasound, magnetic resonance imaging, and computed tomography, and they were examined for any clinical symptoms and signs of the disease. The inclusion criteria were as follows: (1) CRC was confirmed either histologically or using imaging techniques and (2) all patients underwent surgical R0 or R1 resection or chemotherapy using leucovorin/fluorouracil/oxaliplatin (FOLFOX)- or Capoxbased regimens. The exclusion criteria were as follows: (1) had clinical evidence of infection or other inflammatory diseases; (2) at the time of laboratory testing, anticancer treatments had been administered; and (3) patients with primary tumors in other organs We extracted relevant routine laboratory data measured on the day of admission and the clinicopathological data for the selected CRC patients from electronic medical records, including the serum levels of CRP, albumin, and tumor markers of carcinoembryonic antigen (CEA), carbohydrate antigen 19-9 (CA19-9), age, sex, degree of differentiation, tumor location, and tumor node metastasis stage (Amin et al., 2017). Patients underwent complete follow-up after the initial treatment. The start time of the follow-up was the date of the initial diagnosis of CRC and the end time was August 2020 or date of death.

This study was approved by the Local Ethics Committee of the Guangxi Medical University Cancer Hospital, and it was conducted in accordance with the ethical guidelines of the 2008 Declaration of Helsinki and of the current hospital. All patients provided written informed consent. 


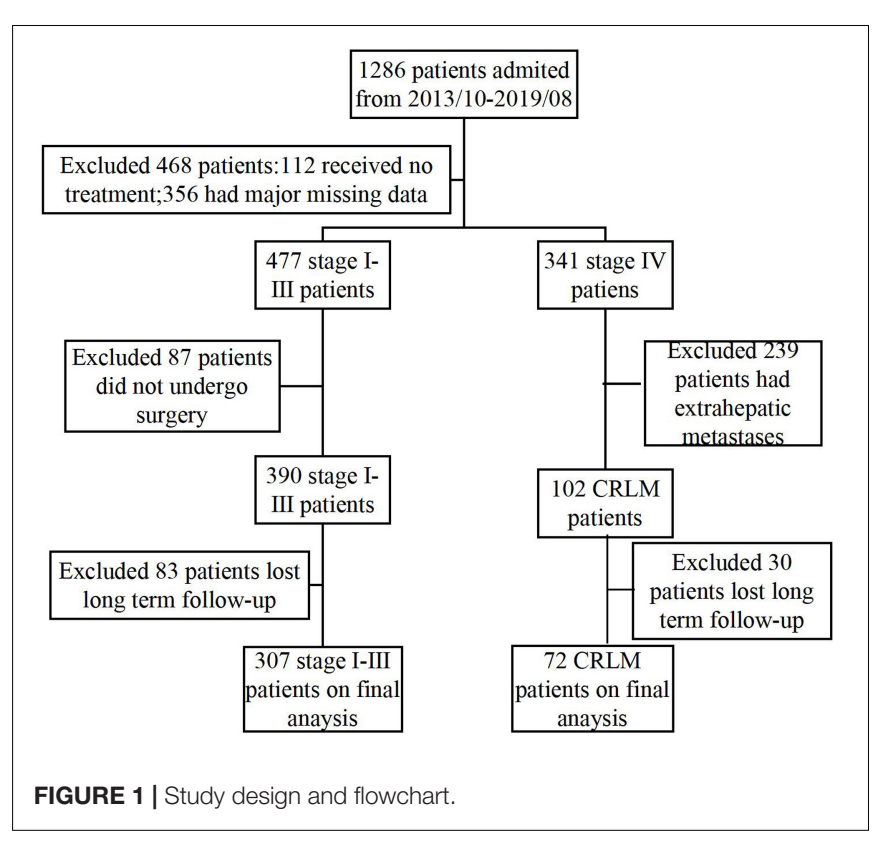

\section{Method for the Detection or Calculation of Each Index}

CRP was detected with the latex enhanced immunoturbidimetric method. Albumin was detected using the bromocresol green method. The levels of serum CEA and CA19-9 were measured with a chemiluminescence microparticle immunoassay using the Arhcitect i2000SR analyzer and the corresponding reagent kits, which were purchased from Architect Diagnostics (Abbott Park, IL, United States). All operations followed the manufacturer's instructions. Systemic inflammatory response was assessed using CRP, GPS, and CAR. GPS is a cumulative score and was calculated as described previously (McMillan, 2013). Patients with both an elevated level of CRP ( $>10 \mathrm{mg} / \mathrm{L})$ and hypoalbuminemia (albumin $<35 \mathrm{~g} / \mathrm{L}$ ) were categorized as GPS2, and those showing one or neither of these blood chemistry abnormalities were categorized as GPS1 or GPS0, respectively. CAR was calculated as CAR $=$ serum CRP level $(\mathrm{mg} / \mathrm{L}) /$ serum albumin level $(\mathrm{g} / \mathrm{L})$.

\section{Statistical Analysis}

Statistical analyses were conducted using SPSS (version 21.0) and $\mathrm{R}$ (version 3.5.1). Data are expressed as the mean \pm standard deviation (SD). The optimal cutoff values were assessed by using receiver operating characteristic (ROC) curves based on the Youden index (YI = sensitivity + specificity -1$)$ and by X-tile software. The end point of survival analysis is OS. Spearman's or Pearson's correlation analysis was used to determine the association between CRP, GPS, and CAR and the other clinicopathological features. Kaplan-Meier analysis and log-rank test were used to determine the differences in the survival curves between the two groups. The ROC curve was constructed to evaluate the discriminative ability of each prognostic score, and the area under the ROC curve (AUC) was measured and compared with that obtained by the method established by DeLong et al. (1988). The Cox proportional hazards model was used for univariate and multivariate analyses to assess potential prognostic factors. A nomogram was formulated using $\mathrm{R}$ version 3.5.1. The discriminative ability of the nomogram was evaluated with the concordance index ( $C$-index). The larger the $C$-index, the more accurate the prognostic prediction. The calibration ability of the nomogram was evaluated by a calibration plot. The closer the calibration curve is to the reference line, the more perfect the calibration. All statistical tests were two-sided, and when the associated probability was less than $0.05, p$-values were considered statistically significant.

\section{RESULTS}

Overall, 379 patients were enrolled (male/female $=250: 129$ ), including 307 stage I-III patients and 72 CRLM patients. The optimal cutoff level for CAR was 0.16 (AUC $=0.753$, sensitivity $=67.82 \%$, specificity $=78.42 \%$ ) for OS in all recruited CRC patients (Figure 2A). For subgroup analysis, the optimal cutoff level for CAR for OS was 0.14 (AUC $=0.713$, sensitivity $=62 \%$, specificity $=75.88 \%)$ in all stage I-III CRC patients (Figure 2B), and that in CRLM patients was 0.25 $($ AUC $=0.786$, sensitivity $=75.68 \%$, specificity $=82.86 \%)$ (Figure 2C). Using the $\mathrm{X}$-tile software, the optimal cutoff levels for CAR in all recruited CRC patients, stage I-III CRC patients, and CRLM patients were $0.64,0.63$, and 0.33 , respectively (Supplementary Figure 1).

\section{Correlation Between the Pretreatment C-Reactive Protein, Glasgow Prognostic Score, and C-Reactive Protein-to-Albumin Ratio and Clinicopathological Features}

The correlation analysis in all CRC patients revealed that pretreatment CRP was positively correlated with sex, location of the primary tumor, pretreatment CEA and CA19-9, lymphatic invasion, and stage and negatively correlated with pathological differentiation. Pretreatment GPS was also revealed to be positively correlated with age, location of the primary tumor, pretreatment CEA and CA19-9, and stage and negatively correlated with pathological differentiation in CRC patients. In addition, pretreatment CAR was positively correlated with sex, location of the primary tumor, pretreatment CEA and CA19-9, and stage and negatively correlated with pathological differentiation in CRC patients (Table 1). In stage I-III CRC patients, the correlation analysis revealed that pretreatment CRP was positively correlated with sex and location of the primary tumor. Also, pretreatment GPS was positively correlated with age and pretreatment CEA. The analysis further revealed that pretreatment CAR was positively correlated with sex and the location of the primary tumor in stage I-III CRC patients (Table 2). The correlation analysis in CRLM patients revealed that pretreatment CRP was positively correlated with the location of the primary tumor, and pretreatment CEA and CA19-9. It was also revealed that pretreatment GPS was 


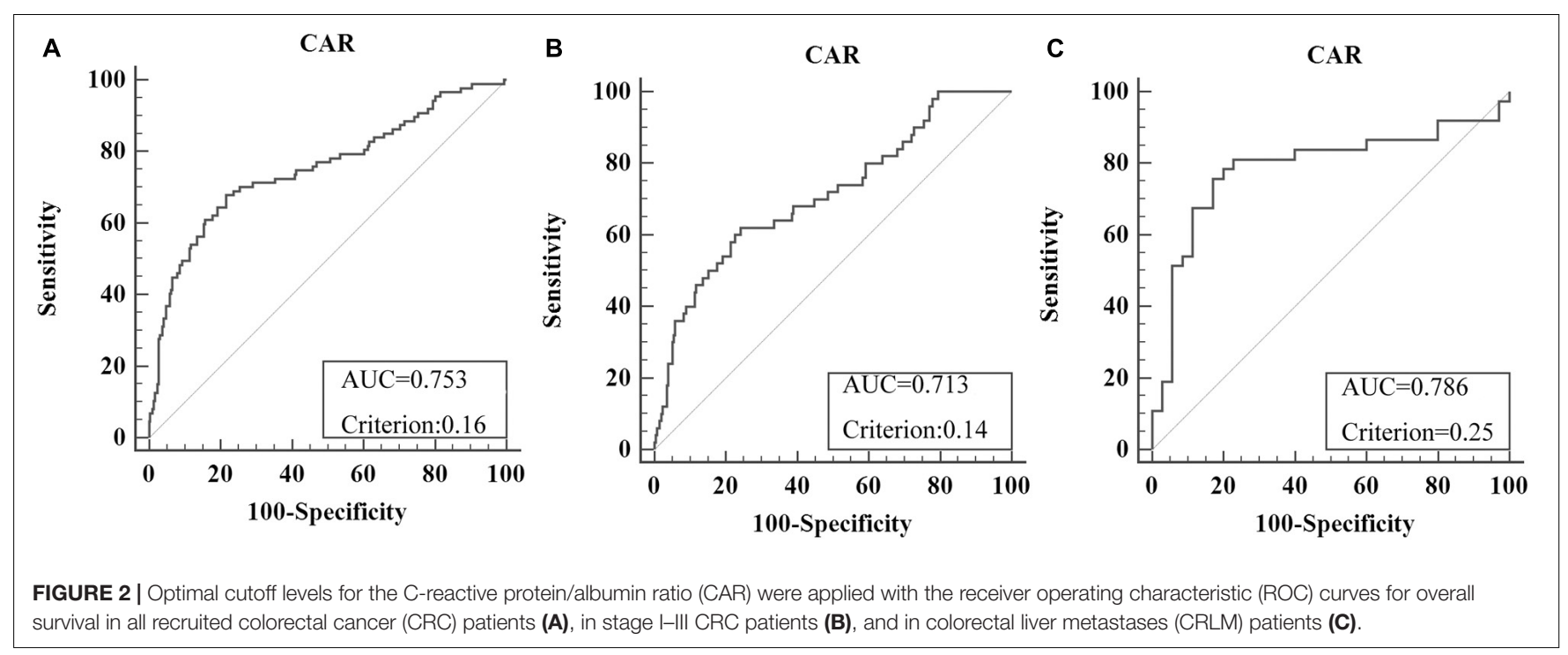

TABLE 1 | Significant correlations of pretreatment CRP, GPS, and CAR with the clinicopathological features in CRC patients.

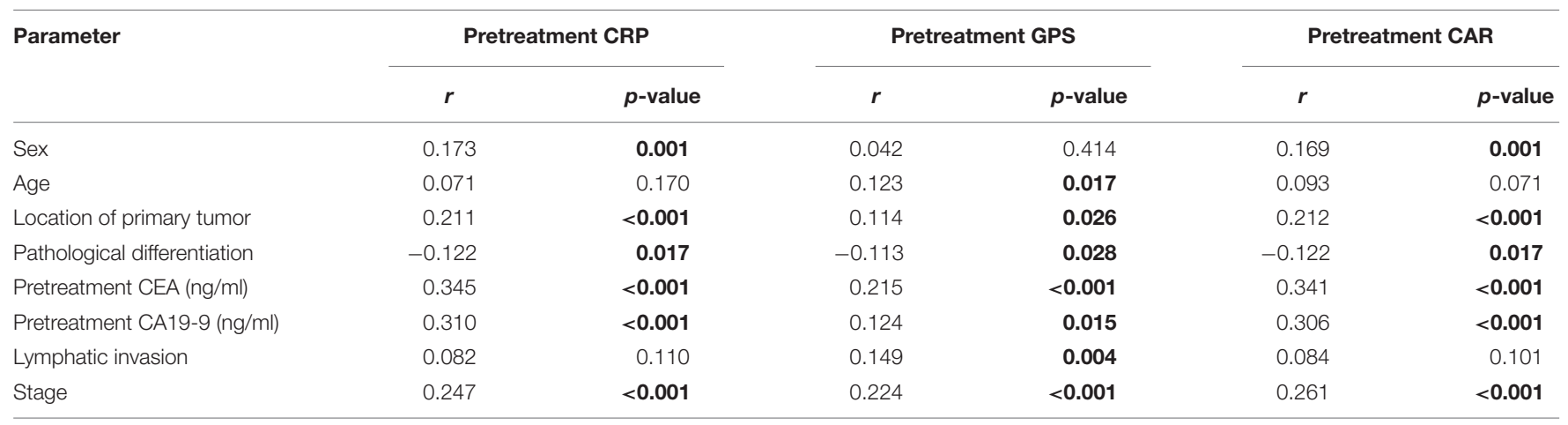

If two variables are continuous, Pearson's correlation analysis was used. If two variables are categorical, or one categorical and one continuous, Spearman's correlation analysis was used. Values in bold indicate statistical significance $(p<0.05)$. CRP, C-reactive protein; GPS, Glasgow Prognostic Score; CAR, CRP-to-albumin ratio; CRC, colorectal cancer; CEA, carcinoembryonic antigen; CA19, carbohydrate antigen 19-9.

TABLE 2 | Significant correlations of pretreatment CRP, GPS, and CAR with the clinicopathological features of stage I-III CRC patients.

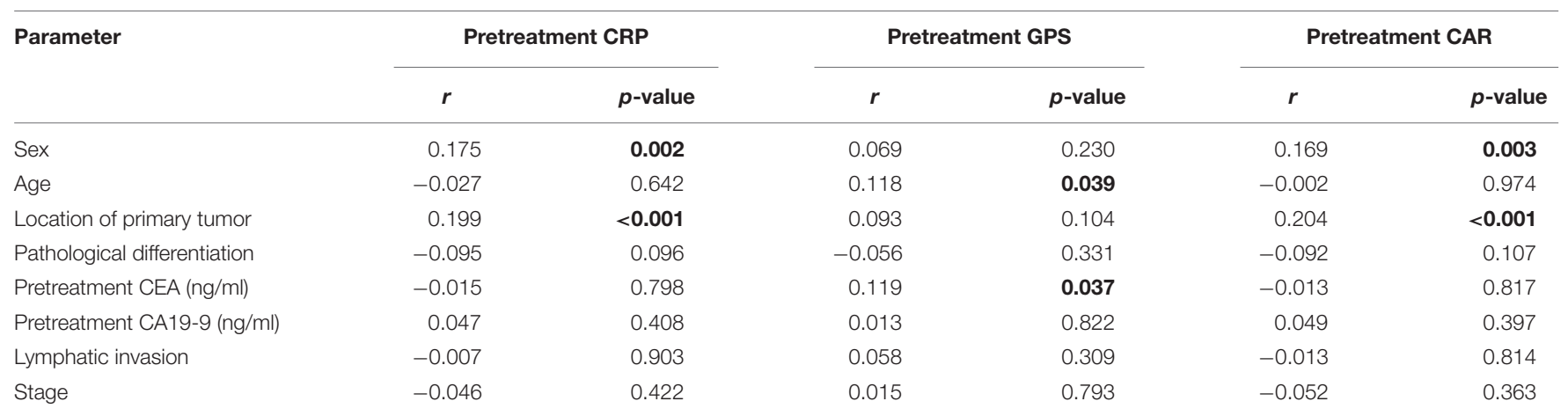

If two variables are continuous, Pearson's correlation analysis was used. If there are two categorical variables, or one categorical and one continuous, Spearman's correlation analysis was used. Values in bold indicate statistical significance $(p<0.05)$. CRP, C-reactive protein; GPS, Glasgow Prognostic Score; CAR, CRP-to-albumin ratio; CRC, colorectal cancer; CEA, carcinoembryonic antigen; CA19, carbohydrate antigen 19-9.

positively correlated with pretreatment CEA and CA19-9. The analysis also showed that pretreatment CAR was positively correlated with pretreatment CEA and CA19-9 in CRLM patients (Table 3).

\section{Survival Analysis Based on Pretreatment Inflammatory Markers}

For CRC patients, Kaplan-Meier analysis and the log-rank tests demonstrated that those with high CRP and GPS had poorer OS 
TABLE 3 | Significant correlations of pretreatment CRP, GPS, and CAR with the clinicopathological features of CRLM patients.

\begin{tabular}{|c|c|c|c|c|c|c|}
\hline \multirow[t]{2}{*}{ Parameter } & \multicolumn{2}{|c|}{ Pretreatment CRP } & \multicolumn{2}{|c|}{ Pretreatment GPS } & \multicolumn{2}{|c|}{ Pretreatment CAR } \\
\hline & $r$ & $p$-value & $r$ & $p$-value & $r$ & $p$-value \\
\hline Sex & 0.039 & 0.748 & -0.094 & 0.431 & 0.035 & 0.774 \\
\hline Age & -0.068 & 0.568 & 0.106 & 0.373 & 0.093 & 0.071 \\
\hline Location of primary tumor & 0.261 & 0.027 & 0.222 & 0.061 & 0.221 & 0.064 \\
\hline Pathological differentiation & -0.100 & 0.401 & -0.142 & 0.233 & -0.074 & 0.539 \\
\hline Pretreatment CEA (ng/ml) & 0.409 & $<0.001$ & 0.346 & 0.003 & 0.390 & $<0.001$ \\
\hline Pretreatment CA19-9 (ng/ml) & 0.367 & 0.002 & 0.380 & 0.001 & 0.349 & 0.003 \\
\hline Lymphatic invasion & -0.054 & 0.653 & 0.082 & 0.496 & -0.019 & 0.876 \\
\hline
\end{tabular}

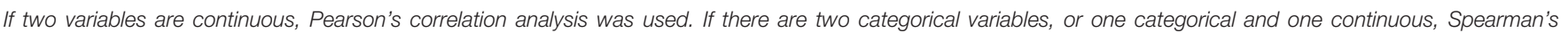

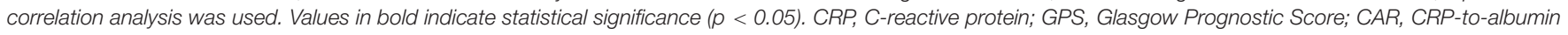
ratio; CRLM, colorectal liver metastases; CEA, carcinoembryonic antigen; CA19, carbohydrate antigen 19-9.

than did those with lower values of the corresponding indices. Kaplan-Meier analysis and log-rank tests also demonstrated that those with high CARs had poorer OS than did patients with lower corresponding values, both in the cohorts of stage I-III patients and CRLM patients $(p<0.05)$ (Supplementary Figures 2, 3).

Kaplan-Meier analysis and the log-rank tests also demonstrated that stage I-III CRC patients with high CRP, GPS, and CAR had poorer OS than those with lower values of the corresponding indices $(p<0.05)$ (Figure 3 ).

In CRLM patients, the results of Kaplan-Meier analysis and the log-rank tests showed that patients with high CRP, GPS, and CAR had poorer OS than those with corresponding lower values $(p<0.05)$ (Figure 4).

\section{Prognostic Value of Outcome Prediction Among the Three Inflammation-Based Prognostic Scores}

ROC curves were used to analyze patient survival at the 24-, 36-, and 48-month follow-up periods, and the AUC assessed the discriminative ability of each inflammation-based prognostic score in CRC patients, stage I-III CRC patients, and CRLM patients (Figure 5). Each prognostic score based on inflammation showed similar discriminative ability. These indicators show better performance in long-term prognostic value for CRLM patients than for stage I-III CRC patients.

\section{Prognostic Factors Influencing Long-Term Survival}

The correlation values between OS and various clinicopathological factors in stage I-III CRC patients are shown in Table 4. In the univariate analysis, OS showed significant relationship with sex, location of the primary tumor, pathological differentiation, lymphatic invasion, pretreatment CEA, CRP, GPS, and CAR, and stage (all $p<0.05$ ). Multivariate analysis indicated that the location of the primary tumor [hazard ratio $(\mathrm{HR})=0.3210,95 \% \mathrm{CI}=0.164-0.627, p=0.001$ ], pathological differentiation $(\mathrm{HR}=0.5014,95 \% \mathrm{CI}=0.258-0.973$, $p=0.041)$, pretreatment CEA ( $\mathrm{HR}=3.1573,95 \% \mathrm{CI}=1.574-$ 6.332, $p=0.001)$, and pretreatment CAR $(\mathrm{HR}=3.9310,95 \%$
$\mathrm{CI}=2.116-7.303, p<0.001)$ were independent prognostic factors for OS. Because CRP, CAR, and GPS contained the same data, three tables were made to consider them separately when performing a multifactor analysis. The HR of CAR was the highest; therefore, the table containing CAR was included in the main manuscript (Table 4); the rest of the results are presented as Supplementary Material (Supplementary Tables 1, 2).

The correlations between OS and the clinicopathological factors in CRLM patients are shown in Table 5. In the univariate analysis, OS was significantly associated with pretreatment CA199, CRP, GPS, and CAR (all $p<0.05$ ). Multivariate analysis indicated that pretreatment CAR $(\mathrm{HR}=5.208,95 \% \mathrm{CI}=2.189$ 12.386, $p<0.001$ ) was an independent prognostic factor for OS. The HR of CAR was the highest; therefore, the table containing CAR was included in the main manuscript (Table 5); the rest of the results are presented as Supplementary Material (Supplementary Tables 3, 4).

The correlations between OS and the clinicopathological factors in CRC patients are shown in Supplementary Table 5. In the univariate analysis, OS showed significant relationship with sex, location of the primary tumor, pathological differentiation, lymphatic invasion, pretreatment CEA, CRP, GPS, and CAR, and stage (all $p<0.05$ ). Multivariate analysis indicated that the location of the primary tumor $(\mathrm{HR}=0.5773,95 \% \mathrm{CI}=0.367-$ $0.909, p=0.018)$, lymphatic invasion $(\mathrm{HR}=2.9439,95 \%$ $\mathrm{CI}=1.664-5.208, p<0.001)$, pretreatment CEA $(\mathrm{HR}=2.2166$, $95 \% \mathrm{CI}=1.304-3.768, p=0.003)$, stage $(\mathrm{HR}=3.4852$, 95\% CI $=2.069-5.872, p<0.001)$, and pretreatment CAR $(\mathrm{HR}=4.3122,95 \% \mathrm{CI}=2.647-7.024, p<0.001)$ were independent prognostic factors for OS. The HR of CAR was the highest; the table containing CAR, CRP, GPS are presented as Supplementary Material (Supplementary Tables 5-7).

\section{Establishing Colorectal Cancer Survival Nomograms Based on the Prognostic Scores of Inflammation}

The prognostic nomogram for OS in CRC patients is shown in Figure 6A. The $C$-index for OS prediction was 0.85 (95\% $\mathrm{CI}=0.80-0.91)$. Figure $6 \mathrm{~B}$ shows the prognostic nomogram for OS in the stage I-III CRC cohort, with a $C$-index for OS 

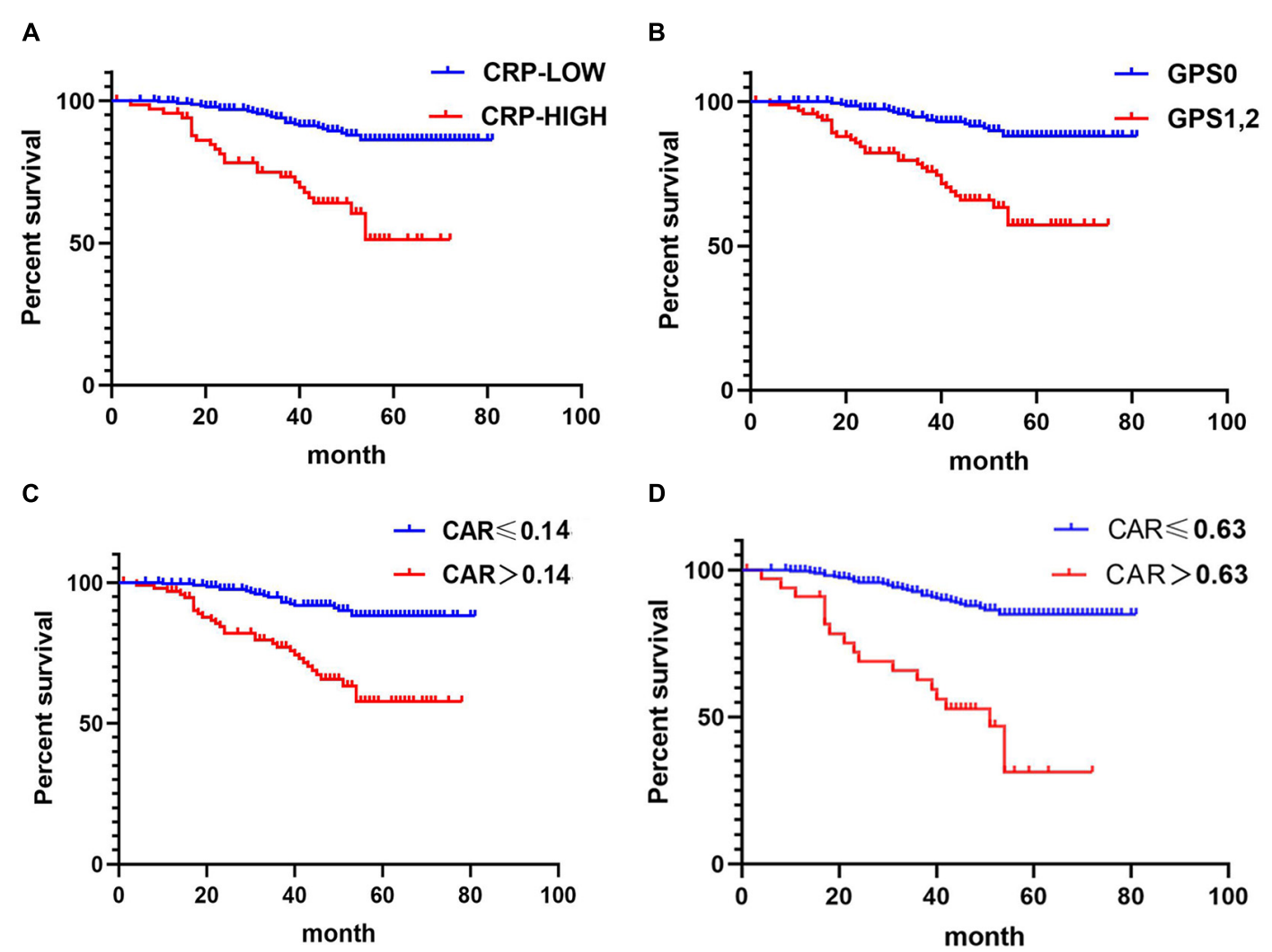

FIGURE 3 | Relationship between C-reactive protein (CRP), CRP-to-albumin ratio (CAR), and Glasgow Prognostic Score (GPS) and overall survival (OS) in patients undergoing surgery for stage I-III colorectal cancer (CRC). (A) Relationship between the two CRP groups and OS. (B) Relationship between the two GPS groups and OS. (C) Relationship between the two CAR groups and OS. CAR was analyzed by grouping based on the cutoff value of the receiver operating characteristic (ROC) analysis in Figure 2B. (D) Relationship between the two CAR groups and OS. The optimal cutoff levels for CAR were produced using the X-tile plot in Supplementary Figure 1B.

prediction of $0.81(95 \% \mathrm{CI}=0.73-0.88)$. For the CRLM cohort, the prognostic nomogram for $\mathrm{OS}$ is shown in Figure 6C. The $C$-index for OS prediction was $0.81(95 \% \mathrm{CI}=0.70-0.93)$. The values of the $C$-index suggest that the nomograms based on the prognostic scores of inflammation were useful predictors for survival of CRC patients. The calibration plot demonstrated a favorable agreement between the predicted and the observed values (Supplementary Figure 4).

\section{DISCUSSION}

CRC is a major health problem worldwide, with 1.8 million new cases and 881,000 deaths a year, accounting for approximately one-tenth of all new cancer cases and deaths (Bray et al., 2018). Over the past 20 years, the United States and Japan have identified patients with early-stage rectal cancer using various examination methods and intervened with the disease as early as possible, effectively reducing the incidence of CRC and the associated mortality (Schreuders et al., 2015). Therefore, effective early diagnosis has an important effect on patient prognosis. Currently, the CEA level is widely used as a prognostic biomarker. However, the sensitivity and specificity of this method are limited in the prognosis of CRC. The identification of new tumor biomarkers is essential to improve the diagnosis and survival of patients.

There is increasing evidence that cancer is related to inflammation. The link between inflammation and cancer is generally such that tumors usually appear in sites of chronic inflammation, and inflammatory cells can be found in tumor biopsy samples (Kinoshita et al., 2013). In addition, recent studies have shown that inflammation was closely related to cancer progression and metastasis. This is probably because the growth or invasion of tumors can cause tissue inflammation, and the production of inflammatory cytokines can, in turn, promote persistent tumor growth, invasion, and metastasis. Furthermore, some studies even suggested that the inflammatory microenvironment could be the seventh sign of cancer (Balkwill and Mantovani, 2001; Qian et al., 2011; Elinav et al., 2013). Therefore, inflammatory markers can predict the prognosis of patients with various cancers, including CRC.

CRP, an inflammatory marker, is an acute-phase reactant synthesized by liver cells (Morris-Stiff et al., 2008). An elevated CRP level reflects the inflammatory response caused by tumor necrosis. Formation of the inflammatory microenvironment is 

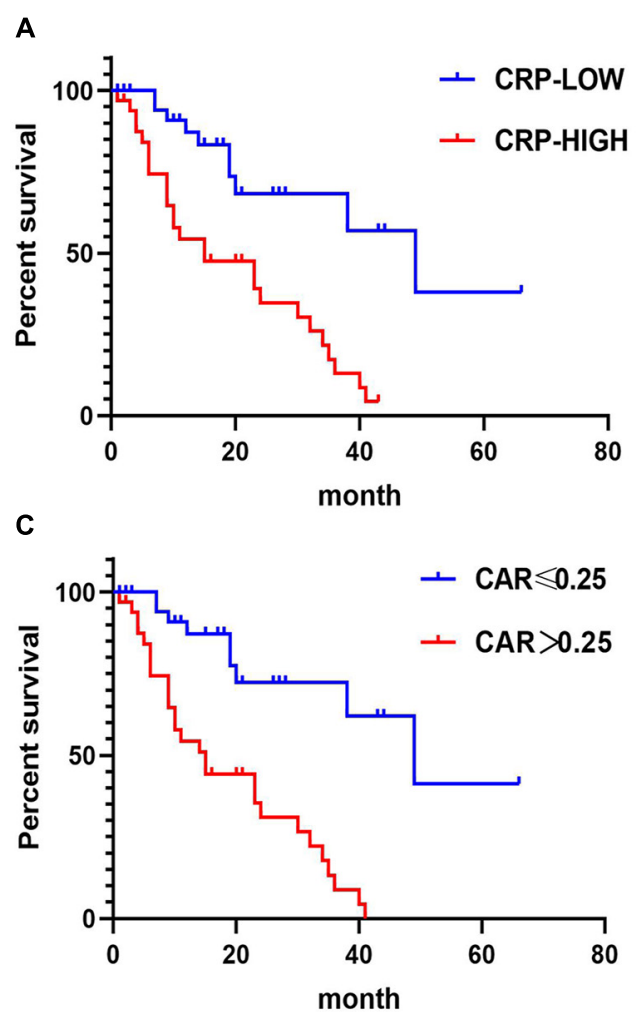

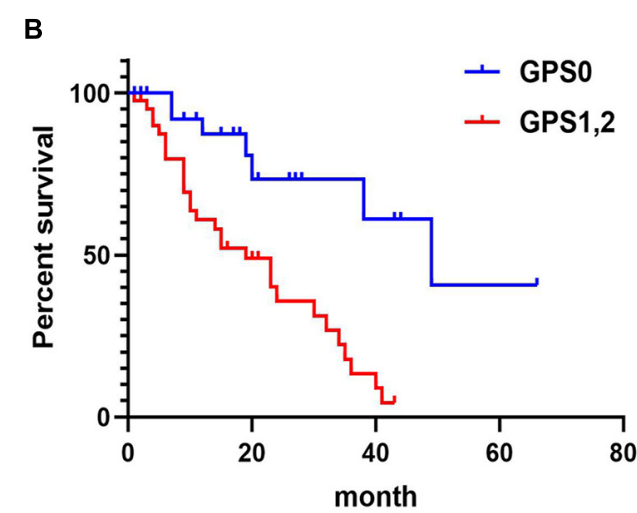

D

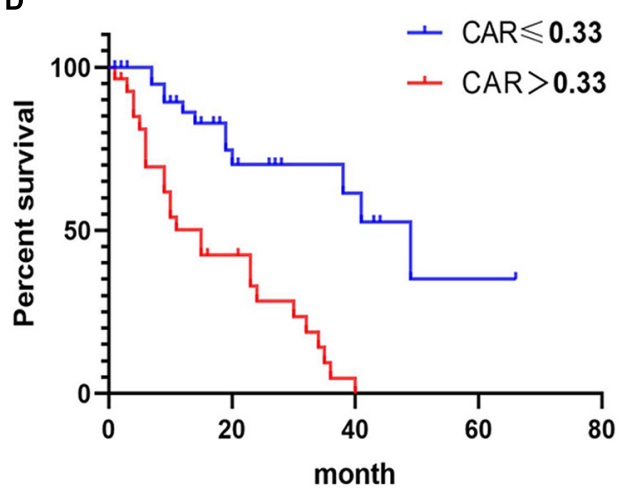

FIGURE 4 | Relationship between C-reactive protein (CRP), CRP-to-albumin ratio (CAR), and Glasgow Prognostic Score (GPS) and overall survival (OS) in patients receiving treatment for colorectal liver metastases (CRLM). (A) Relationship between the two CRP groups and OS. (B) Relationship between the two GPS groups and OS. (C) Relationship between the two CAR groups and OS. CAR was analyzed by grouping based on the cutoff value of the receiver operating characteristic (ROC) analysis in Figure 2C. (D) Relationship between the two CAR groups and OS. The optimal cutoff levels for CAR were produced using the X-tile plot in

Supplementary Figure 1C.

beneficial for persistent tumor growth, invasion, and metastasis (Wong et al., 2007). In this study, CRLM patients had higher CRP levels, which supported this notion, to some extent. Albumin also participates in inflammation. Low albumin levels are associated with poor long-term survival rates in various types of cancer and are more common in patients with advanced cancer (Wang et al., 2019). The prognostic scores of GPS and CAR are based on the CRP and albumin levels, and they may be related to inflammation and poor survival. In the past few years, CRP, GPS, and CAR have been shown to have prognostic value in patients with hepatocellular carcinoma (Kinoshita et al., 2015) and gallbladder (Utsumi et al., 2020), pancreatic (Primavesi et al., 2020), ovarian (Liu et al., 2017), esophageal (Sakai et al., 2020), and gastric (Okugawa et al., 2020) cancers.

This study is the first that directly compares the prognostic value of CRP, GPS, and CAR in patients with primary CRC and liver metastases over multiple time periods. It is interesting to note that these indicators showed better performance in long-term prognostic value for CRLM patients than for stage I-III CRC patients. Pretreatment CRP, GPS, and CAR were independently associated with OS in CRC patients, stage I-III CRC patients, and CRLM patients. Pretreatment CEA was independently associated with the OS of CRC patients and stage I-III CRC patients, while there was no significant relationship between CEA and OS in CRLM patients. However, it is common for CRLM patients to have normal CEA levels. One study showed that approximately 55\% (271/491) of CRC patients undergoing surgery had normal preoperative serum CEA levels (Ishizuka et al., 2012). The above evidence shows that, compared with tumor markers, inflammation-based prognostic systems demonstrate a unique predictive capacity in CRC patients.

Previous studies have reported that the peripheral inflammation markers, including the levels of CRP, GPS, and CAR, are related to the prognosis of CRC patients (Ishizuka et al., 2016; Chen et al., 2017), and this was confirmed in our study. However, results of the subgroup analysis of patients with primary CRC and patients with metastases in the same study have not been reported. As we all know, distant metastasis is one of the important factors affecting the prognosis of patients. Therefore, the results are biased when the analysis for OS was performed on stage I-III patients and patients with distant metastases as a group. Even in studies that have analyzed stage I-III CRC patients or metastatic patients alone (Shibutani et al., 2016a,b; Sakamoto et al., 2020; Son et al., 2020), the optimal cutoff levels of CAR and the ethnicity have been different. Therefore, the prognostic value of CAR 

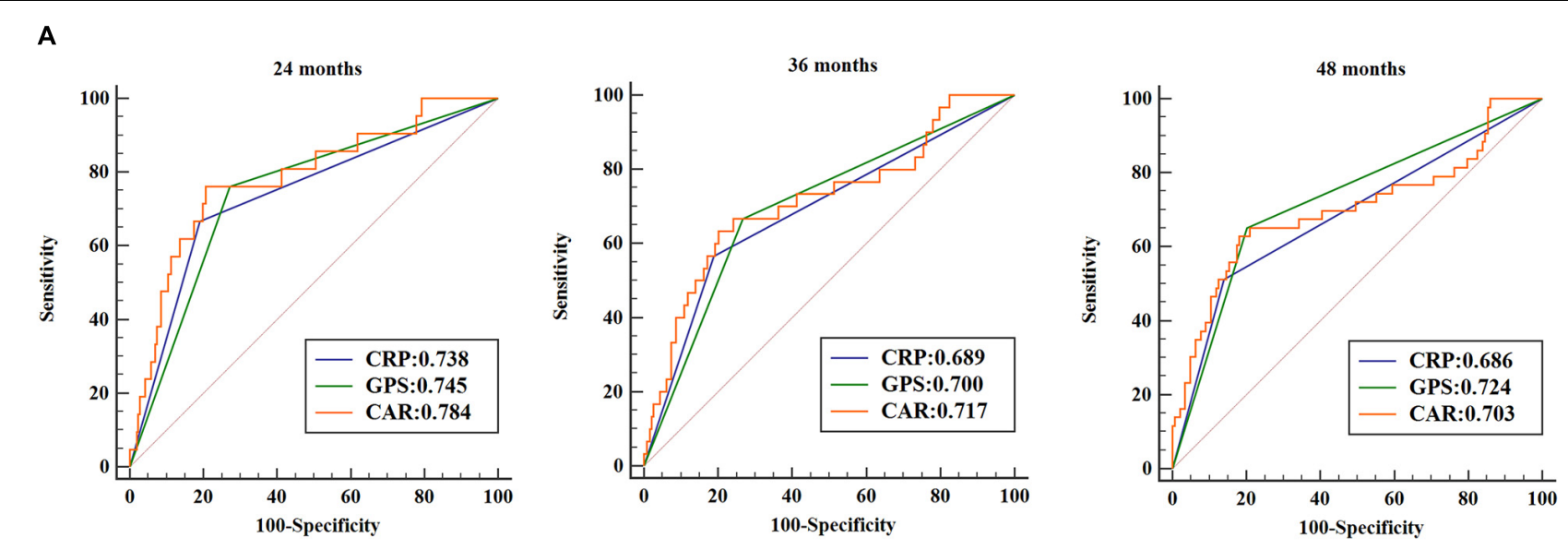

B
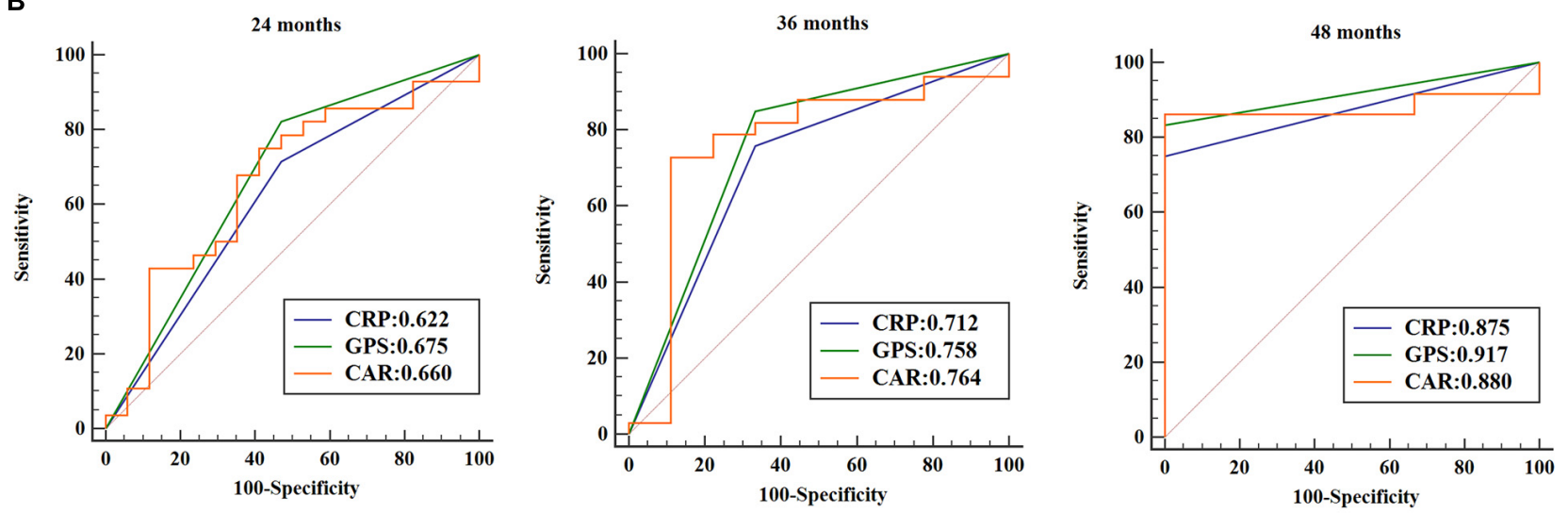

C
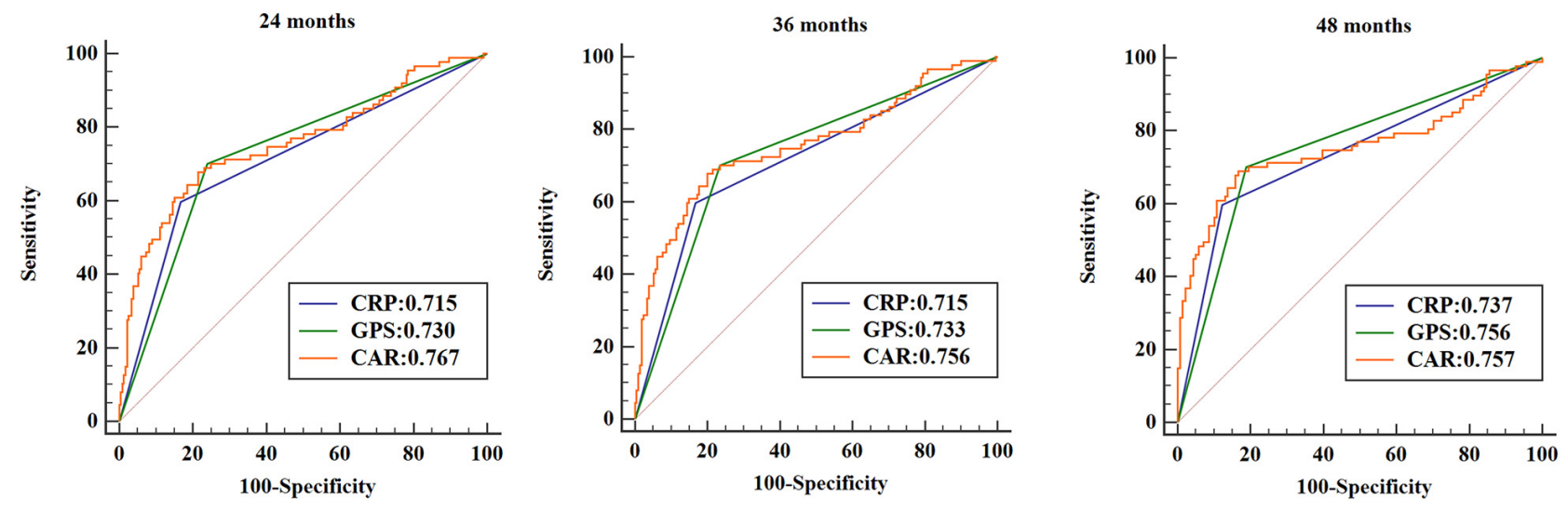

FIGURE 5 | Receiver operating characteristic (ROC) curves were used to analyze patient survival at the 24-, 36-, and 48-month follow-up periods. The areas under the ROC curve (AUCs) assessed the discriminative ability of each inflammation-based prognostic score in stage I-III colorectal cancer (CRC) patients (A), in colorectal liver metastasis (CRLM) patients (B), and in all CRC patients (C).

in patients with CRC cannot be clearly compared between different studies. Racial differences among patients may also contribute to the differences, but the reasons for the differences in outcomes remain unclear. In this study, we tried to ensure the consistency of the study participants in order to explore the most suitable beneficiary group. Patients with stage I-III CRC and patients with liver metastases were discussed separately. This study is helpful for clinicians to detect patients with poor prognosis early and intervene in time so as to prolong their survival time.

Presently, the commonly used methods to determine the optimal cutoff values included the median value method, ROC 
TABLE 4 | Correlations between overall survival and the clinicopathological factors in stage I-III CRC patients.

\begin{tabular}{|c|c|c|c|c|c|c|}
\hline & \multicolumn{3}{|c|}{ Univariate analysis } & \multicolumn{3}{|c|}{ Multivariate analysis } \\
\hline & Hazard ratio & $95 \% \mathrm{Cl}$ & $p$-value & Hazard ratio & $95 \% \mathrm{Cl}$ & $p$-value \\
\hline Sex (female vs. male) & 2.0918 & $1.071-4.086$ & 0.031 & 1.8118 & $0.913-3596$ & 0.089 \\
\hline $\operatorname{Age}(\leq 60$ vs. $>60$ years $)$ & 1.6372 & $0.940-2.850$ & 0.081 & & & \\
\hline Location of primary tumor (colon vs. rectum) & 0.3523 & $0.187-0.663$ & 0.001 & 0.3210 & $0.164-0.627$ & 0.001 \\
\hline Pathological differentiation (poor vs. well, moderate) & 0.4898 & $0.264-0.910$ & 0.024 & 0.5014 & $0.258-0.973$ & 0.041 \\
\hline Lymphatic invasion (ly0 vs. ly1, ly2, ly3) & 3.2600 & $1.779-5.972$ & $<0.001$ & 1.5453 & $0.485-4.923$ & 0.462 \\
\hline Pretreatment CEA ( $\leq 5$ vs. $>5 \mathrm{ng} / \mathrm{ml}$ ) & 4.4961 & $2.302-8.782$ & $<0.001$ & 3.1573 & $1.574-6.332$ & 0.001 \\
\hline Pretreatment CA19-9 ( $\leq 37$ vs. > 37 ng/ml) & 1.6682 & $0.834-3.337$ & 0.148 & & & \\
\hline Pretreatment CRP ( $\leq 10$ vs. > $10 \mathrm{ng} / \mathrm{ml}$ ) & 4.4098 & $2.527-7.696$ & $<0.001$ & & & \\
\hline Pretreatment GPS (0 vs. 1, 2) & 4.6248 & $2.607-8.204$ & $<0.001$ & & & \\
\hline Pretreatment CAR ( $\leq 0.14$ vs. $>0.14)$ & 4.4661 & $2.520-7.917$ & $<0.001$ & 3.9310 & $2.116-7.303$ & $<0.001$ \\
\hline Stage (I, II vs. III) & 2.6337 & $1.487-4.664$ & $<0.001$ & 1.6278 & $0.554-4.784$ & 0.376 \\
\hline
\end{tabular}

Values in bold indicate statistical significance ( $p$ < 0.05). CRC, colorectal cancer; CEA, carcinoembryonic antigen; CA19, carbohydrate antigen 19-9.

TABLE 5 | Correlations between overall survival and the clinicopathological factors in CRLM patients.

\begin{tabular}{|c|c|c|c|c|c|c|}
\hline & \multicolumn{3}{|c|}{ Univariate analysis } & \multicolumn{3}{|c|}{ Multivariate analysis } \\
\hline & Hazard ratio & $95 \% \mathrm{Cl}$ & $p$-value & Hazard ratio & $95 \% \mathrm{Cl}$ & $p$-value \\
\hline Sex (female vs. male) & 0.7009 & $0.342-1.438$ & 0.332 & & & \\
\hline Age ( $\leq 60$ vs. $>60$ years $)$ & 1.6772 & $0.863-3.258$ & 0.127 & & & \\
\hline Location of primary tumor (colon vs. rectum) & 0.9722 & $0.506-1.866$ & 0.932 & & & \\
\hline Pathological differentiation (poor vs. well, moderate) & 1.0564 & $0.461-2.420$ & 0.897 & & & \\
\hline Lymphatic invasion (ly0 vs. ly1, ly2, ly3) & 1.9437 & $0.465-8.125$ & 0.362 & & & \\
\hline Pretreatment CEA ( $\leq 5$ vs. $>5 \mathrm{ng} / \mathrm{ml})$ & 1.0035 & $0.457-2.203$ & 0.993 & & & \\
\hline Pretreatment CA19-9 ( $\leq 37$ vs. > 37 ng/ml) & 2.1406 & $1.087-4.215$ & 0.028 & 1.0387 & $0.503-2.144$ & 0.918 \\
\hline Pretreatment CRP ( $\leq 10$ vs. > $10 \mathrm{ng} / \mathrm{ml}$ ) & 3.8803 & $2.818-8.282$ & $<0.001$ & & & \\
\hline Pretreatment GPS (0 vs. 1, 2) & 4.5748 & $1.889-11.08$ & $<0.001$ & & & \\
\hline Pretreatment CAR ( $\leq 0.25$ vs. $>0.25)$ & 5.3006 & $2.388-11.77$ & $<0.001$ & 5.2076 & $2.189-12.386$ & $<0.001$ \\
\hline
\end{tabular}

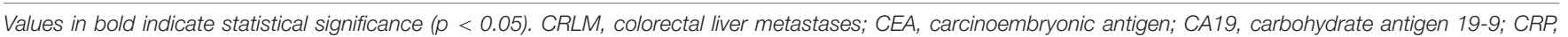
C-reactive protein; GPS, Glasgow Prognostic Score; CAR, CRP-to-albumin ratio.

analysis, and the X-tile software. In this study, in addition to obtaining the optimal cutoff value of CAR through ROC analysis for subsequent analysis, we also used X-tile software to obtain the optimal cutoff value. Kaplan-Meier analysis and the log-rank tests showed that, patients with high CAR values obtained by the two methods have worse OS than those with low values, which scientifically reveals the significance of CAR in the prognosis of CRC patients, both in the stage I-III and CRLM patient cohorts. However, the optimal cutoff value obtained using the X-tile software for stage I-III CRC patients was higher than that for CRLM patients, which is not conducive to clinical application. This may be because there are not enough CRLM patients included.

In this study, CAR was able to accurately divide CRC patients into two independent groups, similarly to CRP and GPS. Because CAR and GPS are based on only two measured parameters and have high AUC values, they can be conventionally available, simple, and inexpensive. Some studies have found that inflammation markers were related to tumor progression (Xu et al., 2015; Ishizuka et al., 2016). We also found that pretreatment CRP, GPS, and CAR were significantly correlated with stage in CRC patients. Therefore, it is possible to determine the optimal cutoff value of CAR for tumors at different stages. However, the best cutoff value for CAR reported in previous studies was different from that obtained in this study, and there was a big gap in the CAR obtained in this study. An accurate determination of the optimal cutoff value of CAR to facilitate the promotion and application of this prognostic index in clinical practice requires further research. GPS is a cumulative scoring method based on a normal reference range. The simplicity and consistency of this indicator makes it very practical for clinical use. Climent et al. (2019) found that the OS difference between the high and low CAR groups was not significant, which is inconsistent with our results. This may be because their research subject was mismatch repair-deficient CRC. To improve the accuracy of prognosis, we have developed survival nomograms based on the three markers, which might increase the clinical significance of the study. The nomograms performed well in predicting survival, and predictions were supported by the $C$-index $(0.85,0.81$, and 0.81 for CRC patients, stage IIII CRC cohort, and the CRLM cohort, respectively) and the calibration plot. 


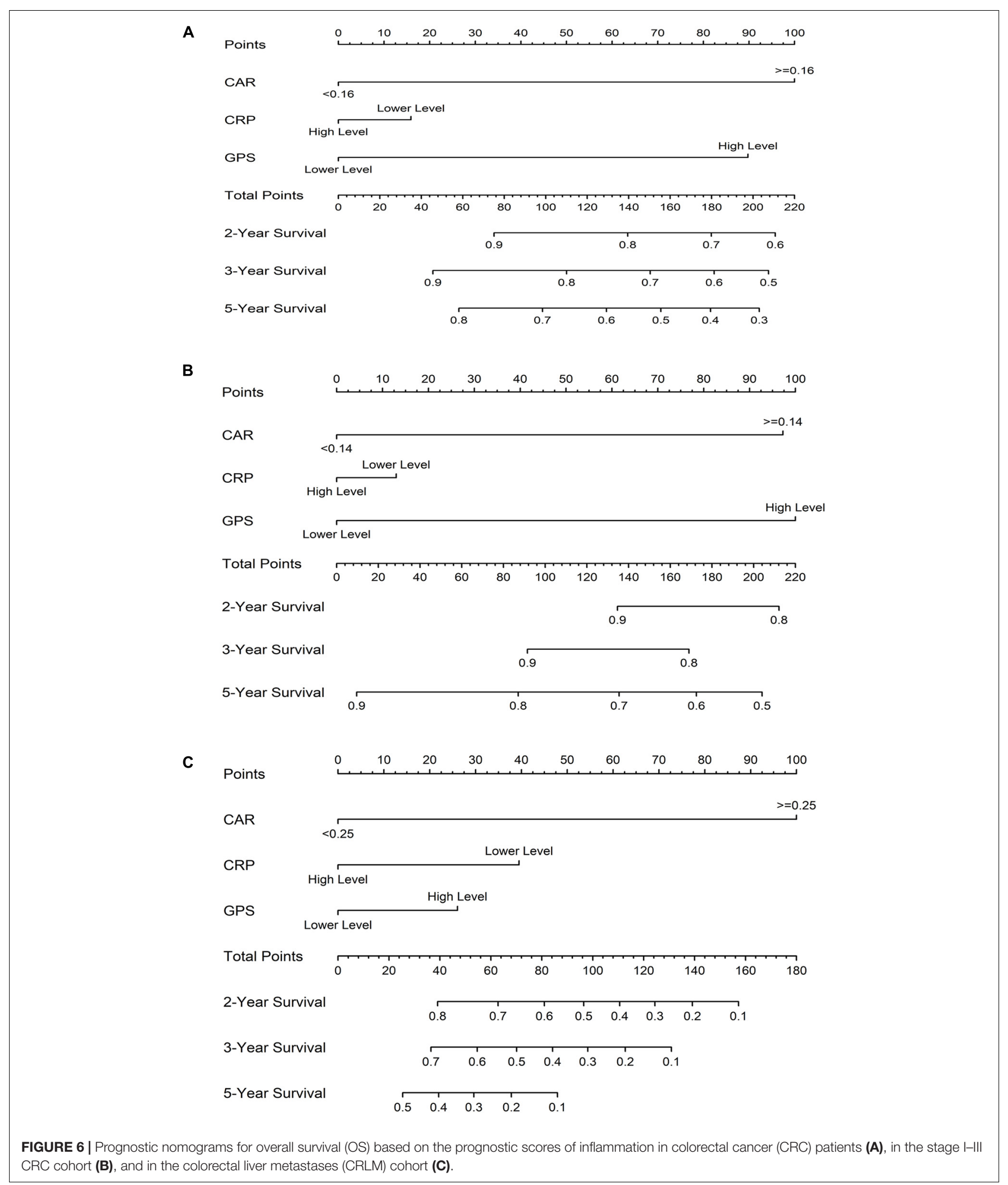

There are some potential limitations to this study. Firstly, this is a retrospective single-center study. Secondly, the same variables (CRP and albumin) were included in the analysis of CRP, GPS, and CAR in our multivariate analysis. Thirdly, the best cutoff value for pretreatment CAR is unknown. Therefore, a large-scale, multicenter prospective study is still necessary in the future. 
In summary, CRC patients with high CRP, GPS and CAR values have worse OS than those with corresponding low values. The three markers have independent prognostic values in patients with CRC, both in the cohorts of stage I-III patients and CRLM patients. Furthermore, we have developed survival nomograms based on the three markers, which might increase the clinical significance of the study.

\section{DATA AVAILABILITY STATEMENT}

The datasets presented in this study can be found in online repositories. The names of the repository/repositories and accession number(s) can be found in the article/ Supplementary Material.

\section{AUTHOR CONTRIBUTIONS}

JZ designed the study, performed the statistical analysis, and prepared the manuscript. JZ, WW, $\mathrm{SN}$, and $\mathrm{BH}$ acquired the data. JL helped with quality control of data and algorithms. JZ and $\mathrm{JL}$ analyzed and interpreted the data. KL and LZ edited the manuscript. All authors reviewed the manuscript and approved the final version.

\section{REFERENCES}

Amin, M., Greene, F., Edge, S., Compton, C., Gershenwald, J., Brookland, R., et al. (2017). The Eighth edition AJCC cancer staging manual: continuing to build a bridge from a population-based to a more "personalized" approach to cancer staging. CA Cancer J. Clin. 67, 93-99. doi: 10.3322/caac. 21388

Balkwill, F., and Mantovani, A. (2001). Inflammation and cancer: back to Virchow? Lancet 357, 539-545. doi: 10.1016/s0140-6736(00)04046-0

Bray, F., Ferlay, J., Soerjomataram, I., Siegel, R., Torre, L., and Jemal, A. (2018). Global cancer statistics 2018: GLOBOCAN estimates of incidence and mortality worldwide for 36 cancers in 185 countries. CA Cancer J. Clin. 68, 394-424. doi: $10.3322 /$ caac. 21492

Chen, Y., Zhang, J., Zhang, W., Yang, Z., Luo, R., and Kang, S. (2017). [C-reactive protein/albumin ratio as a novel inflammation-based prognostic index for predicting outcomes of patients with colorectal cancer]. Nan Fang Yi Ke Da Xue Xиe Bao 37, 622-627.

Climent, M., Ryan, É, Stakelum, Á, Khaw, Y., Creavin, B., Lloyd, A., et al. (2019). Systemic inflammatory response predicts oncological outcomes in patients undergoing elective surgery for mismatch repair-deficient colorectal cancer. Int. J. Colorectal Dis. 34, 1069-1078. doi: 10.1007/s00384-019-03274-6

DeLong, E., DeLong, D., and Clarke-Pearson, D. (1988). Comparing the areas under two or more correlated receiver operating characteristic curves: a nonparametric approach. Biometrics 44, 837-845. doi: 10.2307/2531595

Elinav, E., Nowarski, R., Thaiss, C., Hu, B., Jin, C., and Flavell, R. (2013). Inflammation-induced cancer: crosstalk between tumours, immune cells and microorganisms. Nat. Rev. Cancer 13, 759-771. doi: 10.1038/nrc3611

Hamilton, T., Leugner, D., Kopciuk, K., Dixon, E., Sutherland, F., and Bathe, O. (2014). Identification of prognostic inflammatory factors in colorectal liver metastases. BMC Cancer 14:542. doi: 10.1186/1471-2407-14-542

Haruki, K., Shiba, H., Horiuchi, T., Sakamoto, T., Gocho, T., Fujiwara, Y., et al. (2017). Impact of the C-reactive protein to albumin ratio on long-term outcomes after hepatic resection for colorectal liver metastases. Am. J. Surg. 214, 752-756. doi: 10.1016/j.amjsurg.2017.02.001

Ishizuka, M., Nagata, H., Takagi, K., Iwasaki, Y., and Kubota, K. (2012). Inflammation-based prognostic system predicts postoperative survival of

\section{FUNDING}

This study was supported by grants from the Natural Science Foundation of Guangxi (Nos. 2017GXNSFAA198155 and 2018GXNSFBA281049), Guangxi Scientific Research \& Technical Planning Project (Guike AB19110018), the Scientific Research \& Technical Development Project of Qingxiu District, Nanning City, Guangxi Province (Nos. 2016051, 2017034, and 2017036), and Shenzhen Key Medical Discipline Construction Fund (No. SZXK053).

\section{ACKNOWLEDGMENTS}

This work was supported by the Shenzhen Public Service Platform on Tumor Precision Medicine and Molecular Diagnosis.

\section{SUPPLEMENTARY MATERIAL}

The Supplementary Material for this article can be found online at: https://www.frontiersin.org/articles/10.3389/fcell.2021. 637650/full\#supplementary-material

colorectal cancer patients with a normal preoperative serum level of carcinoembryonic antigen. Ann. Surg. Oncol. 19, 3422-3431. doi: 10.1245/ s10434-012-2384-5

Ishizuka, M., Nagata, H., Takagi, K., Iwasaki, Y., Shibuya, N., and Kubota, K. (2016). Clinical significance of the C-reactive protein to albumin ratio for survival after surgery for colorectal cancer. Ann. Surg. Oncol. 23, 900-907. doi: 10.1245/s10434-015-4948-7

Kinoshita, A., Onoda, H., Imai, N., Iwaku, A., Oishi, M., Tanaka, K., et al. (2013). The glasgow prognostic score, an inflammation based prognostic score, predicts survival in patients with hepatocellular carcinoma. BMC Cancer 13:52. doi: 10.1186/1471-2407-13-52

Kinoshita, A., Onoda, H., Imai, N., Iwaku, A., Oishi, M., Tanaka, K., et al. (2015). The C-reactive protein/albumin ratio, a novel inflammation-based prognostic score, predicts outcomes in patients with hepatocellular carcinoma. Ann. Surg. Oncol. 22, 803-810. doi: 10.1245/s10434-014-4048-0

Køstner, A., Kersten, C., Löwenmark, T., Ydsten, K., Peltonen, R., Isoniemi, H., et al. (2016). The prognostic role of systemic inflammation in patients undergoing resection of colorectal liver metastases: C-reactive protein (CRP) is a strong negative prognostic biomarker. J. Surg. Oncol. 114, 895-899. doi: $10.1002 /$ jso. 24415

Liu, Y., Chen, S., Zheng, C., Ding, M., Zhang, L., Wang, L., et al. (2017). The prognostic value of the preoperative c-reactive protein/albumin ratio in ovarian cancer. BMC Cancer 17:285. doi: 10.1186/s12885-017-3220-x

Mantovani, A., Allavena, P., Sica, A., and Balkwill, F. (2008). Cancer-related inflammation. Nature 454, 436-444. doi: 10.1038/nature07205

Matsubara, D., Arita, T., Nakanishi, M., Kuriu, Y., Murayama, Y., Kudou, M., et al. (2020). The impact of postoperative inflammation on recurrence in patients with colorectal cancer. Int. J. Clin. Oncol. 25, 602-613. doi: 10.1007/s10147019-01580-1

McMillan, D. (2013). The systemic inflammation-based glasgow prognostic score: a decade of experience in patients with cancer. Cancer Treat. Rev. 39, 534-540. doi: 10.1016/j.ctrv.2012.08.003

McMillan, D., Crozier, J., Canna, K., Angerson, W., and McArdle, C. (2007). Evaluation of an inflammation-based prognostic score (GPS) in patients undergoing resection for colon and rectal cancer. Int. J. Colorectal Dis. 22, 881-886. doi: 10.1007/s00384-006-0259-6 
Morris-Stiff, G., Gomez, D., and Prasad, K. (2008). C-reactive protein in liver cancer surgery. Eur. J. Surg. Oncol. 34, 727-729. doi: 10.1016/j.ejso.2008.01.016

Nasr, R., Salim Hammoud, M., Nassar, F., Mukherji, D., Shamseddine, A., and Temraz, S. (2018). Inflammatory markers and microRNAs: the backstage actors influencing prognosis in colorectal cancer patients. Int. J. Mol. Sci. 19:1867. doi: 10.3390/ijms19071867

Okugawa, Y., Toiyama, Y., Yamamoto, A., Shigemori, T., Ichikawa, T., Yin, C., et al. (2020). Lymphocyte-to-C-reactive protein ratio and score are clinically feasible nutrition-inflammation markers of outcome in patients with gastric cancer. Clin. Nutr. 39, 1209-1217. doi: 10.1016/j.clnu.2019.05.009

Primavesi, F., Andreasi, V., Hoogwater, F., Partelli, S., Wiese, D., Heidsma, C., et al. (2020). A preoperative clinical risk score including C-reactive protein predicts histological tumor characteristics and patient survival after surgery for sporadic non-functional pancreatic neuroendocrine neoplasms: an international multicenter cohort study. Cancers 12:1235. doi: 10.3390/ cancers 12051235

Qian, B., Li, J., Zhang, H., Kitamura, T., Zhang, J., Campion, L., et al. (2011). CCL2 recruits inflammatory monocytes to facilitate breast-tumour metastasis. Nature 475, 222-225. doi: 10.1038/nature 10138

Sakai, M., Sohda, M., Saito, H., Ubukata, Y., Nakazawa, N., Kuriyama, K., et al. (2020). Comparative analysis of immunoinflammatory and nutritional measures in surgically resected esophageal cancer: a single-center retrospective study. In vivo 34, 881-887. doi: 10.21873/invivo.11853

Sakamoto, Y., Mima, K., Imai, K., Miyamoto, Y., Tokunaga, R., Akiyama, T., et al. (2020). Preoperative C-reactive protein-to-albumin ratio and clinical outcomes after resection of colorectal liver metastases. Surg. Oncol. 35, 243-248. doi: 10.1016/j.suronc.2020.09.014

Schreuders, E., Ruco, A., Rabeneck, L., Schoen, R., Sung, J., Young, G., et al. (2015). Colorectal cancer screening: a global overview of existing programmes. Gut 64, 1637-1649. doi: 10.1136/gutjnl-2014-309086

Shibutani, M., Maeda, K., Nagahara, H., Iseki, Y., Hirakawa, K., and Ohira, M. (2016a). The significance of the C-reactive protein to albumin ratio as a marker for predicting survival and monitoring chemotherapeutic effectiveness in patients with unresectable metastatic colorectal cancer. SpringerPlus 5:1798. doi: 10.1186/s40064-016-3529-y

Shibutani, M., Maeda, K., Nagahara, H., Iseki, Y., Ikeya, T., and Hirakawa, K. (2016b). Prognostic significance of the preoperative ratio of c-reactive protein to albumin in patients with colorectal cancer. Anticancer Res. 36, 995-1001.
Son, W., Shin, S., Park, S., Lee, S., Park, E., Baik, S., et al. (2020). Clinical impact of combined modified glasgow prognostic score and c-reactive protein/albumin ratio in patients with colorectal cancer. Diagnostics 10:859. doi: 10.3390/ diagnostics10110859

Utsumi, M., Aoki, H., Nagahisa, S., Nishimura, S., Une, Y., Kimura, Y., et al. (2020). Preoperative C-reactive protein/albumin ratio as a predictive factor for gallbladder carcinoma. In Vivo 34, 1901-1908. doi: 10.21873/invivo.11986

Wang, Y., Liu, Z., Xu, D., Liu, M., Wang, K., and Xing, B. (2019). Fibrinogen-albumin ratio index (FARI): a more promising inflammationbased prognostic marker for patients undergoing hepatectomy for colorectal liver metastases. Ann. Surg. Oncol. 26, 3682-3692. doi: 10.1245/s10434-01907586-3

Wong, V., Malik, H., Hamady, Z., Al-Mukhtar, A., Gomez, D., Prasad, K., et al. (2007). C-reactive protein as a predictor of prognosis following curative resection for colorectal liver metastases. Br. J. Cancer 96, 222-225. doi: 10.1038/ sj.bjc. 6603558

Xu, X., Yu, H., Hu, W., Song, Q., and Mao, W. (2015). A novel inflammation-based prognostic score, the C-reactive protein/albumin ratio predicts the prognosis of patients with operable esophageal squamous cell carcinoma. PLoS One 10:e0138657. doi: 10.1371/journal.pone.0138657

Conflict of Interest: The authors declare that the research was conducted in the absence of any commercial or financial relationships that could be construed as a potential conflict of interest.

Publisher's Note: All claims expressed in this article are solely those of the authors and do not necessarily represent those of their affiliated organizations, or those of the publisher, the editors and the reviewers. Any product that may be evaluated in this article, or claim that may be made by its manufacturer, is not guaranteed or endorsed by the publisher.

Copyright (C) 2021 Zhou, Wei, Hou, Ning, Li, Huang, Liu and Zhang. This is an open-access article distributed under the terms of the Creative Commons Attribution License (CC BY). The use, distribution or reproduction in other forums is permitted, provided the original author(s) and the copyright owner(s) are credited and that the original publication in this journal is cited, in accordance with accepted academic practice. No use, distribution or reproduction is permitted which does not comply with these terms. 